\title{
Modelo explicativo sobre trayectorias vitales y desarrollo vocacional en deportistas de alto nivel Explanatory model on personal path and vocational development in high level athletes Pedro Ricardo Álvarez-Pérez, David López-Aguilar Universidad de La Laguna(España)
}

\begin{abstract}
Resumen. El objetivo de este artículo es presentar un modelo explicativo acerca del desarrollo vocacional y la construcción del proyecto profesional y vital de los deportistas de alto nivel. A partir de una revisión teórica, se integran diferentes factores y competencias en un modelo sistémico y holístico, que tiene en cuenta los distintos roles que asume el deportista de alto nivel a lo largo de su trayectoria vital y con el que se pretende orientar y facilitar los procesos de desarrollo integral, tanto en el plano deportivo, como en el plano personal y profesional. Para contrastar la relevancia del modelo teórico y la validez de los diferentes constructos que se contemplan en relación al desarrollo vocacional, se llevó a cabo un estudio descriptivo a través de la aplicación de un cuestionario con una muestra de 189 deportistas de alto nivel reconocidos por el Consejo Superior de Deportes, perteneciente al Ministerio de Educación, Cultura y Deporte del Gobierno de España. Los resultados confirmaron que los procesos de definición y construcción vocacional del deportista de alto nivel tienen una naturaleza multifactorial. En concreto, las variables personales y contextuales tienen una fuerte incidencia en la identidad vocacional del deportista. Asimismo, la participación en experiencias deportivas y académicas, contribuyen a la adquisición de determinadas competencias, habilidades y conocimientos que son de especial interés para la vida en sociedad y para el desarrollo profesional. No obstante, el deportista de alto nivel se encuentra ante serias dificultades para mantener una relación efectiva entre estudios y deporte. Palabras clave: modelo socio-cognitivo; deportistas de alto nivel; proyecto profesional y vital; trayectorias vitales del deportista; modelo sociocognitivo.
\end{abstract}

Abstract. The objective of this article is to present an explanatory model on vocational development and the construction of professional and vital paths in high level athletes. Based on a theoretical review, different factors and competences are integrated into a systemic and holistic model, which takes into account the different roles that high level athletes assume throughout his or her life history. These roles are then used to guide and ease athletes' processes of integral development in the areas of sport, personal life, and profession. To contrast the relevance of the theoretical model and the validity of the different constructs contemplated in the vocational development, a descriptive study was carried out applying a questionnaire to a sample of 189 high level athletes recognized by the Superior Council of Sports, an organ integrated into the Ministry of Education, Culture, and Sport of Spain. The results confirm that vocational definition and construction processes of high level athletes have a multifactorial nature. Specifically, personal and contextual variables have a strong impact on athletes' vocational identity. Likewise, participation in sports and academic experiences contributes to the acquisition of specific competences, skills, and knowledge that are of special interest for social life and for professional development. However, high level athletes are facing serious difficulties in maintaining an effective relationship between studies and sport.

Keywords: socio-cognitive model; High level athletes; Professional and vital project; Vital trajectories of the athlete; Socio-cognitive model.

\section{Introducción}

Son diversos los enfoques y modelos que se han propuesto en las últimas décadas, con la finalidad de ofrecer una explicación teórica y empírica de la conducta y comportamiento vocacional de las personas (Ginzberg, Ginsburg, Axelrad \& Herma, 1951; Roe, 1954; Super, Crites, Hummel, Moser, et al., 1957; Tiedeman \& O’hara, 1963; Holland, 1973; Krumboltz, 1979; etc). Se trata de teorías que han servido de base para elaborar modelos explicativos en relación al desarrollo vocacional y la construcción del proyecto profesional y vital. Entre estos enfoques teóricos de referencia, uno de los más valorados, tanto en el ámbito de la investigación como de la intervención, ha sido el denominado modelo cognitivo social del desarrollo de la carrera profesional (career development) (Lent \& Hackett, 1987; Lent, Brown \& Hackett, 1994; Lent, Brown \& Hackett, 2000; Lent, Hill \& Hoffman, 2003). Los conceptos y constructos nucleares de este enfoque, ofrecen una base sólida a partir de la cual se puede diseñar un modelo explicativo específico centrado en la definición de la identidad vocacional y la construcción del proyecto profesional y vital de los deportistas de alto nivel.

Los modelos diseñados y que se han venido aplicando hasta el momento, atienden a situaciones y condiciones genéricas de los individuos, sin profundizar en variables específicas de relevancia para los deportistas de alto nivel. Esta realidad justifica la necesidad de definirun modelo integral y constructivo, adaptado a las características particulares e individuales de este colectivo. Las peculiares características que presentan los deportistas de alto nivel y la diversidad de situaciones a las que tienen que enfrentarse a lo largo de su trayectoria vital, hace necesario que se disponga de referentes para una intervención adaptada a sus características y necesidades específicas. Entre otras intervenciones, se viene reclamando un apoyo adecuado que facilite la compatibi-

Fecha recepción: 07-07-17. Fecha de aceptación: 13-09-17 Pedro Álvarez Pérez palvarez@ull.es lidad de diversas actividades y la participación en procesos formativos, ya que constituye una base fundamental para el desarrollo de su carrera profesional.

Por este motivo, el objetivo principal de este trabajo es proponer un modelo explicativo sobre el desarrollo vocacional de los deportistas de alto nivel, construido a partir de los fundamentos e ideas principales de los modelos teóricos actuales sobre el desarrollo profesional. De manera específica, se trata de una propuesta que sienta sus bases en algunos constructos como la autoeficacia, expectativas de resultados o el sistema de metas propuesto por el modelo cognitivo social del desarrollo de la carrera. A estos referentes, se añaden otros constructos que aportan otras teorías para explicar, de manera precisa, el proceso de desarrollo vocacional de los deportistas de alto nivel.

Este modelo explicativo podría servir como referente para diseñar programas educativos y orientadores para deportistas de alto nivel, de tal manera que en su trayectoria vital, dispongan de la información, de las condiciones y de las ayudas necesarias para construir su identidad vocacional y un proyecto profesional y vital que esté en consonancia con sus competencias, habilidades y conocimientos, al mismo tiempo que con sus expectativas personales.

\section{Fundamentación teórica del modelo}

Hasta el momento, el fenómeno del desarrollo vocacional de los deportistas de alto nivel ha sido definido desde diversos planteamientos, distintas corrientes teóricas y diferentes disciplinas. En este sentido, resulta necesario combinar teorías, modelos, constructos y conceptos que están próximos al desarrollo vocacional y que sirven para ofrecer una explicación más detallada sobre cómo se produce el proceso de madurez vocacional y cómo se construye el proyecto profesional y vital de los deportistas de alto nivel.

Efectivamente, el modelo que aquí se presenta surge de la conjugación de diferentes conceptos y constructos elaborados a partir de corrientes teóricas diversas, que pueden contribuir a la definición de un 
modelo acerca del desarrollo vocacional de los deportistas de alto nivel más preciso y comprensivo. Se trata de un modelo interdisciplinar cuyo objetivo fundamental es ofrecer una explicación sobre el desarrollo vocacional y la construcción del proyecto profesional y vital de los deportistas de alto nivel que atienda a una perspectiva integral, holística y globalizada.

El referente principal de este modelo es el enfoque cognitivo social definido por Lent, Brown \& Hackett (1994) y Lent, Brown \& Hackett (2000). En términos generales, esta propuesta teórica plantea, por un lado, que el ser humano es un ser activo que tiene capacidad para regular su propia conducta (Valiente, 2000) y, por otro, que el desarrollo vocacional se modula a partir de la combinación de variables contextuales (sociedad, economía, política, etc.) y cognitivas (motivación, realización, emoción, etc.).

El atributo central sobre el que gira el enfoque cognitivo social es el concepto de autoeficacia, entendido como la capacidad que tiene el individuo para evaluar en qué medida, las competencias y conocimientos que posee, le permitirán alcanzar determinados niveles de rendimiento en una acción concreta. Blanco (2009, p.424) considera que el objetivo que tiene la autoeficacia para el individuo es responder a la pregunta: «¿realmente soy capaz de hacerlo?».

Otro importante componente de este enfoque son las expectativas de resultado. Este constructo ha sido definido por Lent, Brown \& Hackett (1994) como el ejercicio que realiza la persona para analizar los posibles resultados que se podrían alcanzar al ejecutar una acción concreta. La autoeficacia y las expectativas de resultado dan lugar al sistema de metas que, desde la perspectiva de estos autores, incluye la definición de los intereses, objetivos y acciones del proyecto vital y profesional de la persona.

Por tanto, este enfoque propone que la definición de los intereses y motivaciones personales se construyen a partir de la autoeficacia y las expectativas de resultado del individuo (figura I). En concreto, se trata de un proceso de identificación en el que las personas y, en este caso concreto, los deportistas desarrollan «interés por una actividad cuando se perciben competentes para su desempeño (expectativas positivas de autoeficacia) y cuando anticipan que de la implicación en la misma se derivarán resultados considerados valiosos (expectativas positivas de resultado)» (Blanco, 2009, p.427).

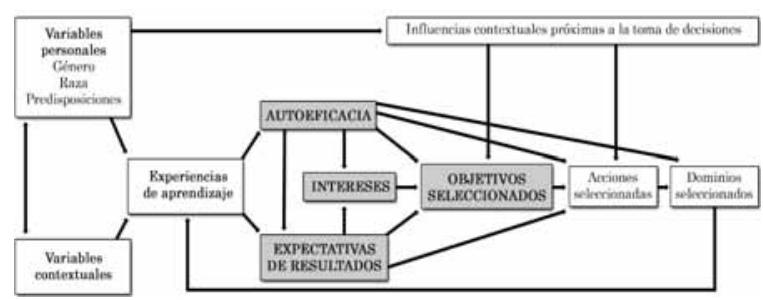

Figura I. Modelo cognitivo social del desarrollo de intereses y toma de decisiones. Adaptado de Lent, Brown \& Hackett (1994, p.43) y Lent, Brown \& Hackett (2000, p.37).

Un rasgo característico y esencial del enfoque cognitivo social es que sitúa al deportista ante un incesante estado de análisis, en el que se replantea si las metas y los logros alcanzados se corresponden con la autoeficacia y las expectativas de resultados proyectados. Es decir, el deportista valora la efectividad de la actividad por la que ha mostrado interés, reconduciendo su revisión a los conceptos de autoeficacia y expectativas de resultados mostrados inicialmente.

En este proceso de autoanálisis, en el que se enfrenta lo deseable (expectativas de resultado) con lo probable (autoeficacia), resulta necesario que el deportista de alto nivel emplee lo que Savickas (2005) y Hartung, Porfeli \& Vondracek (2008) han definido como competencias de adaptabilidad. Este tipo de competencias hacen referencia a la capacidad que tiene el individuo para activar determinadas tareas con la finalidad de planificar, definir y reconducir los proyectos de vida, así como facilitar los procesos de adaptación y ajuste a los entornos profesionales, personales, deportivos, familiares, etc. En concreto, estos autores sostienen que una persona que posee un adecuado perfil de adap- tabilidad, es aquella que tiene un alto nivel de dominio de las dimensiones y competencias que se relacionan en la siguiente tabla.

Tabla 1

Dimensiones y competencias de a daptabilidad. Elaborado a partir de Savickas (2005) \& Savickas y Porfeli (2012)

\begin{tabular}{|c|c|c|}
\hline Dimensión & Definición & $\begin{array}{c}\text { Competencia } \\
\text { asociada }\end{array}$ \\
\hline $\begin{array}{l}\text { eocupación por } \\
\text { el futuro }\end{array}$ & $\begin{array}{l}\text { Hace referencia al sentimiento que tiene el individuo por } \\
\text { prepararse para el futuro y por desarrollar la capacidad de } \\
\text { planificación. }\end{array}$ & Planificación \\
\hline Autocontrol & $\begin{array}{l}\text { Es la creencia que tiene el sujeto de poder regular la } \\
\text { construcción y definición de su propia carrera, que es } \\
\text { responsable de tomar decisiones de manera autónoma y } \\
\text { de manejar adecuadamente los problemas con los que se } \\
\text { encuentra. }\end{array}$ & $\begin{array}{l}\text { Toma de } \\
\text { decisiones }\end{array}$ \\
\hline $\begin{array}{l}\text { Curiosidad por la } \\
\text { exploración de } \\
\text { posibles escenarios }\end{array}$ & $\begin{array}{l}\text { Motiva al individuo a conocer mejor el entorno en el que } \\
\text { se desenvuelve, permitido así un mejor ajuste entre lo que } \\
\text { espera conseguir y lo que realmente es capaz de hacer. }\end{array}$ & Exploración \\
\hline $\begin{array}{l}\text { Confianza para } \\
\text { plantearse retos y } \\
\text { superar obstáculos }\end{array}$ & $\begin{array}{l}\text { Implica la actividad de habilidades y capacidades para } \\
\text { resolver un problema concreto con ciertas garantías de } \\
\text { éxito. }\end{array}$ & $\begin{array}{l}\text { Resolución de } \\
\text { problemas }\end{array}$ \\
\hline
\end{tabular}

Si bien los atributos básicos del enfoque cognitivo social ofrecen una explicación sobre el desarrollo vocacional del individuo, fundamentada en la prevención y caracterizada por ser un proceso que ocurre a lo largo de la vida y que tiene en consideración las variables personales y contextuales, las renovadas teorías de la orientación vocacional y profesional alertan de la necesidad de introducir la perspectiva holística que plantea el marco conceptual denominado life-designing (Savickas, Nota, Rossier, Dauwalder et al., 2009).

De la misma manera, en la literatura especializada sobre el análisis del desarrollo de la carrera deportiva existen multitud de trabajos cuya finalidad prioritaria ha sido definir la trayectoria del deportista de alto nivel (Wylleman, Vanden, De Knop, Sloore et al, 1995; Wylleman \& De Knop, 1997; Wylleman, De Knop, Ewing \& Cumming, 2000). Sin embargo, la mayoría de estos trabajos han sido cuestionados por centrarse exclusivamente en el ámbito deportivo, dejando a un lado otros factores personales que ocupan un importante lugar en la vida del deportista, como pueden ser la familia, el trabajo, la formación, etc. Por ello, como reclama Álvarez (2011, p.117), resulta de interés que, «el deportista de élite, además de como deportista, debe ser visto y tratado como una persona con un desarrollo psicológico, psico-social y académico vocacional».

Precisamente Wylleman \& Lavalle (2004) presentan un modelo sobre el desarrollo de la carrera deportiva que ha sido ampliamente compartido por la comunidad científica (Díaz, Buceta \& Bueno, 2004; Torregrosa, Sánchez \& Cruz, 2004; Wylleman, Alfermann \& Lavallee, 2004; González \& Bedoya, 2008; González \& Torregrosa, 2009) y cuya estructura se asienta sobre una perspectiva global, integradora y holística. En concreto, este modelo de desarrollo integral trata al deportista de alto nivel como una persona que practica deporte, al mismo tiempo que tiene un desarrollo madurativo, social, académico y vocacional (tabla 2).

Tabla 2.

Desarrollo integral de l deportista (Extraído y adaptado de Wylleman \& Lavallee, 2004).

\begin{tabular}{|c|c|c|c|c|c|c|}
\hline & \multicolumn{6}{|c|}{ Edad } \\
\hline & 10 & 15 & 20 & 25 & 30 & 35 \\
\hline $\begin{array}{l}\text { Desarrollo } \\
\text { deportivo }\end{array}$ & Iniciación & Desarrollo & Maes & tría/Perfecci & amiento & $\begin{array}{c}\text { Retirada } \\
\text { Abandono }\end{array}$ \\
\hline $\begin{array}{l}\text { Desarrollo } \\
\text { madurativo }\end{array}$ & Infancia & Adolescenc & & Juventud & & adulta \\
\hline $\begin{array}{l}\text { Desarrollo } \\
\text { Social }\end{array}$ & $\begin{array}{c}\text { Padres } \\
\text { Hermanos } \\
\text { Amigos }\end{array}$ & $\begin{array}{c}\text { Amigos } \\
\text { Entrenado } \\
\text { Padres }\end{array}$ & & $\begin{array}{c}\text { Pareja } \\
\text { Entrenador }\end{array}$ & & nilia \\
\hline $\begin{array}{l}\text { Desarrollo } \\
\text { académico y } \\
\text { vocacional }\end{array}$ & $\begin{array}{l}\text { Educación } \\
\text { primaria }\end{array}$ & $\begin{array}{l}\text { Educación } \\
\text { secundaria }\end{array}$ & & $\begin{array}{l}\text { ucación } \\
\text { uperior }\end{array}$ & $\begin{array}{l}\text { Formac } \\
\text { Ocupac }\end{array}$ & $\begin{array}{l}\text { vocacional } \\
\text { profesional }\end{array}$ \\
\hline
\end{tabular}

La incorporación de esta visión comprensiva al sistema de construcción vocacional del individuo, matiza la definición de los elementos básicos del enfoque cognitivo social. Es decir, la autoeficacia, las expectativas de resultado y la concreción de los intereses y motivaciones personales vienen determinados, además de por variables de tipo personal y contextual, por los distintos roles que han ocupado la vida del deportista de alto nivel (trabajador, estudiante, deportista, miembro de familia, ciudadano, etc.). De este modo, participar en distintos contextos de aprendizaje aporta al deportista experiencias, conocimientos, 
competencias y habilidades que, en última instancia, le permiten construir, de manera más precisa y madura, su identidad vocacional y su proyecto profesional y vital.

Y aunque todos los planos de desarrollo propuestos en el modelo de Wylleman \& Lavallee(2004) favorecen la definición de la autoeficacia y las expectativas de resultado de los deportistas de alto nivel, es la formación y las experiencias deportivas las que contribuyen sobremanera a la construcción de su propia identidad vocacional. No obstante, las investigaciones realizadas hasta el momento, llaman la atención acerca de las dificultades para mantener una relación efectiva entre estudios y deporte (Guille, Sarrazin, Carperten, Trouilloud et al., 2002; Marsh \& Kleiman, 2003; Urbina \& Miranda, 2004; Grissom, 2005; García, Leo, Martín \& Sánchez, 2008; Ruiz, Salinero \& Sánchez, 2008).

Las dificultades para armonizar el deporte con otras actividades, como puede ser la formación, hace que en muchas ocasiones los deportistas de alto nivel encuentren en el abandono una salida para liberarse de la presión y el estrés que provoca la simultaneidad de actividades (Fernández, Stephan \& Fouquereau, 2006; González, Álvarez, Cabrera y Bethencourt, 2007; Álvarez y López, 2012; Álvarez et al., 2014). En consecuencia, y como plantean Garcés \& Carlin (2011, p.36),

«estas contradicciones entre la participación deportiva y otros aspectos de la vida no deportiva, pueden resultar en los deportistas en forma de falta de competencias y habilidades necesarias para lograr éxito en los campos del deporte y en sus carreras post-deportivas».

Las dificultades a las que se enfrenta el deportista de alto nivel para conciliar estudios y deporte, están motivadas por un problema básico y fundamental: la falta de tiempo (García, 1990; Danish, Petitpas \& Hale, 1993; Torregrosa \& Mimbrero, 1998; Wylleman \& Lavallee, 2004; Nuviala \& Nuviala, 2005; Puig \& Vilanova, 2006; Macarro, Romero \& Torres, 2010; Álvarez y López, 2012; Álvarez et al., 2014; López, Barriopedro, Conde, Sánchez et al., 2015). Es decir, mientras que, por un lado, la asistencia regular a clase, la entrega de trabajos y tareas, la realización de exámenes, etc. constituyen las características básicas de la formación reglada, por otro, la asistencia a los entrenamientos, la participación en competiciones, las concentraciones deportivas, etc. son aspectos fundamentales de la vida diaria de los deportistas de alto nivel. Según Torregrosa, Sánchez \& Cruz (2004, p.220), «estas dificultades son atribuidas sistemáticamente por los deportistas a la falta de flexibi- lidad del sistema (...) y la poca consideración que (...) se tiene con los deportistas de alto nivel». Ante esta realidad, los deportistas se ven forzados a priorizar entre estas dos actividades.

De cualquier manera, y teniendo en cuenta que formación y deporte constituyen dos importantes escenarios de aprendizaje, que ayudan al deportista de alto nivel a definir sus intereses vocacionales y a construir su proyecto de vida y, por extensión, a prepararse para su futuro desarrollo profesional, se deberían implantar sistemas de apoyo, cuyos objetivos prioritarios estuvieran centrados en facilitar los procesos de compatibilización entre estudios y deporte y dar respuesta a las necesidades y características particulares de los deportistas de alto nivel (Márquez, Salguero \& Tuero, 2003; Puig \& Vilanova, 2006; García, Leo, Martín \& Sánchez, 2008; Álvarez y López, 2012; Álvarez et al., 2014; Solà, 2015). Por ello, no se debe perder de vista que el deportista de alto nivel

«ha de formarse más y mejor y, como quiera que los cambios en la sociedad tecnológica que le ha tocado vivir son constantes y cada vez más acelerados, habrá de adoptar, además, una actitud variable hacia las demandas de especialización y, consecuentemente, de formación que las relaciones laborales le hacen cada día» (Bermejo, 2006, p.23).

Modelo explicativo sobre el desarrollo vocacional de los deportistas de alto nivel

La aportación de los diferentes componentes teóricos analizados, permitieron diseñar el modelo que se detalla en la figura II. Se trata de una propuesta que defiende que el desarrollo vocacional constituye un recorrido biográfico amplio que se extiende a lo largo de la vida, en el que el deportista se encuentra ante un continuo estado de valoración introspectivo, en el que revisa y replantea, de manera incesante, su proyecto profesional y de vida.

Este análisis sitúa al deportista ante una realidad en la que tiene que enfrentar lo que considera que es capaz de hacer (autoeficacia) y lo que le gustaría llegar a conseguir (expectativas de resultado). Este desafío entre lo probable y lo deseable se construye a partir de dos importantes hitos que tienen lugar en la trayectoria vital del deportista: las experiencias deportivas y las experiencias de aprendizaje en las que participa a lo largo de la vida (educación formal y no formal) Es decir, la actividad

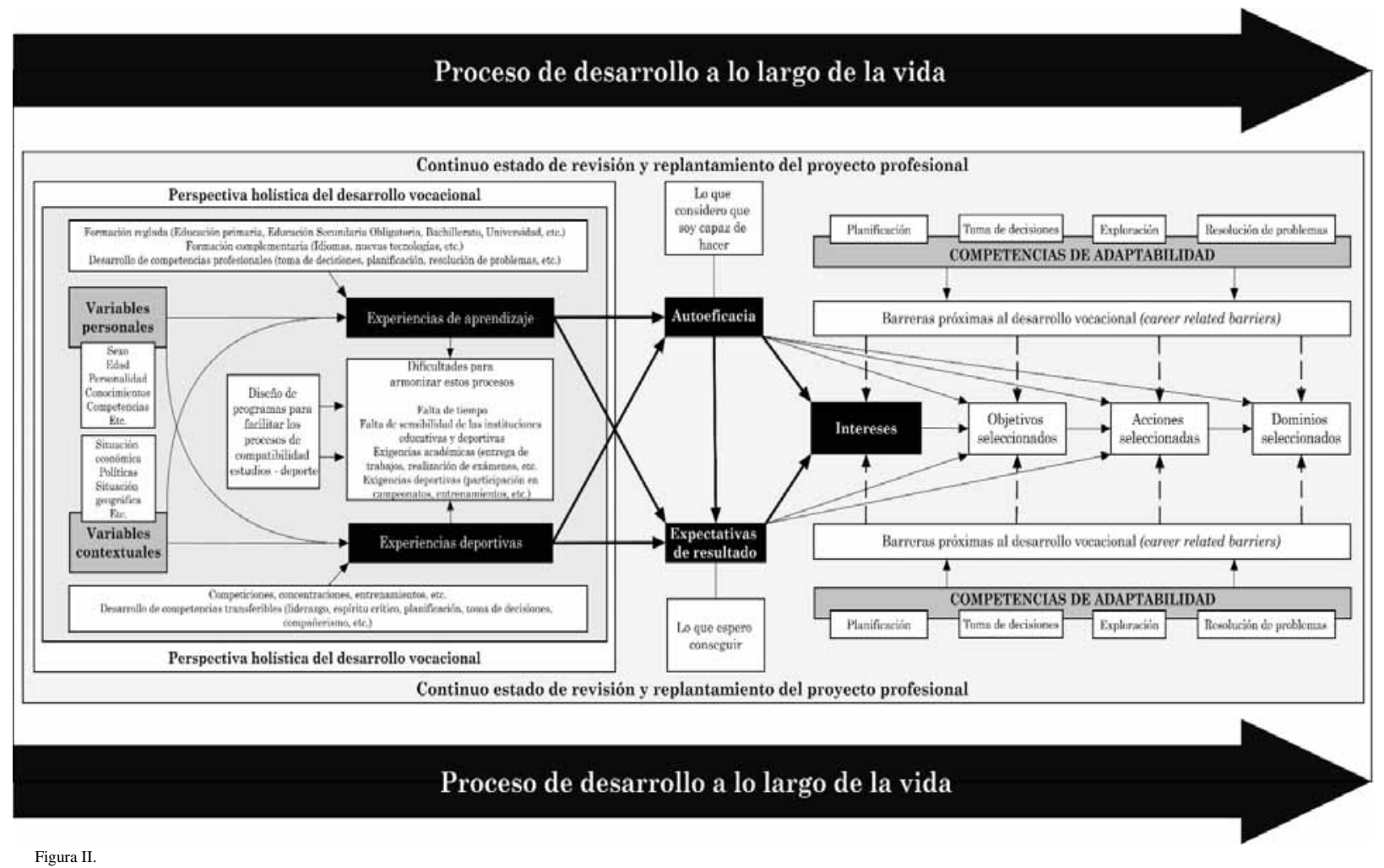

Modelo del desarrollo vocacional de los deportistas de alto nivel 
deportiva y las experiencias de aprendizaje contribuyen tanto al crecimiento personal, como al desarrollo de competencias, conocimientos y habilidades de interés para el desarrollo de su carrera profesional.

La definición de la autoeficacia y de las expectativas de resultado, son la consecuencia directa de una valoración general y holística en la que entran en juego las variables definidas. Es decir, estos dos conceptos nucleares del modelo se construyen a partir de las experiencias vitales que han ocupado al deportista en su recorrido particular, atendiendo al plano formativo, deportivo, social, profesional, contextual y personal.

Todo este proceso y recorrido previo, sitúa al deportista ante una situación en la que identifica sus intereses y motivos vocacionales de una manera meditada y con madurez, donde identifica los objetivos a alcanzar, las acciones a realizar y los conocimientos a dominar para lograr las metas propuestas. A pesar de que este proceso lleva al deportista a replantearse de manera continuada sus intereses y motivaciones personales, es importante no olvidarse de las barreras que encuentran para lograr sus metas profesionales. Por ello, el deportista debe activar las competencias de adaptabilidad que le permitan superar los problemas y dificultades que surjan en su recorrido vocacional.

En la figura II se sintetizan las variables y elementos que, de manera interrelacionada, ayudan a comprender cómo tiene lugar el desarrollo vocacional de los deportistas de alto nivel. El siguiente paso es valorar la relevancia del modelo y de los distintos elementos que lo componen, a través de un estudio con una muestra de deportistas de alto nivel.

\section{Material y método}

Con el fin de comprobar que el sistema conceptual y los componentes incluidos en el modelo explicativo sobre el desarrollo vocacional y la construcción del proyecto profesional y vital definido se justificaba empíricamente, se llevó a cabo un estudio con una muestra de deportistas de alto nivel. En concreto, los participantes en este estudio debían ser deportistas de alto nivel reconocidos por el Consejo Superior de Deportes perteneciente al Ministerio de Educación, Cultura y Deporte del Gobierno de España que, en el momento de administración de las pruebas se encontraran activamente en el mercado laboral, a fin de valorar aspectos relacionados con el desarrollo vocacional y la construcción del proyecto profesional y vital incluidos en el modelo explicativo.

De manera detallada, la muestra que participó en el estudio fue de 189 deportistas de alto nivel, con una edad media de 31,98 años, de los cuales el 67,7\% eran hombres y el 32,3\% mujeres. En cuanto a su formación académica, el 45,2\% había finalizado sus estudios preuniversitarios y el $54,8 \%$ los universitarios. Uno de los aspectos que se cuidó de manera detallada en el proceso de selección muestral fue que hubiera representatividad de todas las comunidades autónomas de España (tabla 3).

\begin{tabular}{|c|c|c|}
\hline \multicolumn{3}{|c|}{ Número total de deportistas de alto nivel participantes en el estudio $=189(n=189)$} \\
\hline Edad & \multirow{2}{*}{\multicolumn{2}{|c|}{$\begin{array}{l}\text { Rango de Edad: } 17-58 ; M=31.98 \text { años; } S d=9.642 \text { años } \\
\text { Hombres }=128(67.7 \%) \text { Mujeres= } 61(32.3 \%)\end{array}$}} \\
\hline Sexo & & \\
\hline \multirow{9}{*}{ Comunidad autónoma } & Madrid $=15.3 \%(\mathrm{n}=29)$ & Castilla y Léon $=4.2 \%(\mathrm{n}=8)$ \\
\hline & Canarias $=13.2 \%(\mathrm{n}=25)$ & País Vasco $=3.2 \%(\mathrm{n}=6)$ \\
\hline & Cataluña $=12.7 \%(\mathrm{n}=24)$ & Baleares $=1.6 \%(\mathrm{n}=3)$ \\
\hline & Valencia $=12.7 \%(n=24)$ & Extremadura $=1.6 \%(\mathrm{n}=3)$ \\
\hline & Andalucía $=7.9 \%(\mathrm{n}=15)$ & Murcia $=1.6 \%(n=3)$ \\
\hline & Galicia $=7.4 \%(n=14)$ & Aragón $=1.1 \%(\mathrm{n}=2)$ \\
\hline & Cantabria $=6.9 \%(\mathrm{n}=13)$ & Asturias $=1.1 \%(\mathrm{n}=2)$ \\
\hline & Navarra $=4.8 \%(\mathrm{n}=9)$ & La Rioja $=0.5 \%(n=1)$ \\
\hline & Castilla La Mancha=4.2\% $(n=8)$ & \\
\hline Estudios cursados & \multicolumn{2}{|c|}{ Estudios preuniversitarios (incluida Formación Profesional): $45.2 \%$} \\
\hline
\end{tabular}

La recogida de información se llevó a cabo a través de un cuestionario elaborado ad hoc que atendía a las características de la muestra a la que iba dirigida y a los objetivos que se pretendían dar respuesta. En concreto, se empleó el «Cuestionario de planificación y definición del Proyecto Personal y Vital de los Deportistas de alto Nivel». Durante el proceso de construcción del instrumento y con la finalidad de asegurar su fiabilidad y validez, se llevaron a cabo distintos procedimientos propuestos por McMillan y Schumacher (2005). En concreto, se realizó una prueba piloto $(n=15)$, una prueba de forma $(n=3)$ y una prueba de contenido $(\mathrm{n}=2)$. Con este proceso se pretendió depurar algunos aspectos referidos al contenido, redacción, comprensión de las preguntas, alternativas de respuesta, pertinencia del contenido, claridad e interés del instrumento, adecuación de las preguntas a los objetivos de la investigación, etc.

Tras incorporar las modificaciones sugeridas por las pruebas, el cuestionario definitivo quedó configurado en torno a las siguientes dimensiones objeto de análisis: datos de identificación (1), proceso de toma de decisiones (2), motivos e intereses profesionales (3), centralidad y finalidad del trabajo (4), planificación del futuro laboral (5), compatibilidad entre deporte y otras actividades (6) y procesos de adaptabilidad (7). En cuanto a la fiabilidad del instrumento, se obtuvo a partir de la prueba Alfa de Cronbach que arrojó un valor elevado: $\alpha=.911$.

El tratamiento de los datos obtenidos se llevó a cabo con el apoyo metodológico del software Statistical Package for the Social Sciences (SPSS 21.0) para el sistema operativo Microsoft Windows 7. De acuerdo con Etxeberria \& Tejedor (2005), los exámenes estadísticos realizados permitieron realizar un análisis descriptivo de los datos obtenidos: medidas de centralización y dispersión.

\section{Resultados}

De acuerdo al modelo explicativo propuesto, los resultados del estudio realizado pusieron de manifiesto que la práctica deportiva de alto nivel era un importante escenario formativo que permitió a los deportistas adquirir competencias y conocimientos transferibles, que eran claves para su desarrollo vocacional y para su ejercicio profesional $(\mathrm{M}=3,10 ; \mathrm{sd}=, 814)$.

En concreto, los encuestados manifestaron que, a través de la práctica deportiva de alto nivel desarrollaron algunas habilidades transferibles, como la toma de decisiones ( $\mathrm{M}=3,46$; $\mathrm{sd}=, 688$ ), la comunicación eficaz $(\mathrm{M}=3,48$; $\mathrm{sd}=, 606)$, la resolución de problemas $(\mathrm{M}=3,35 \mathrm{sd}=, 680)$ o la planificación del tiempo $(\mathrm{M}=3,43$; $\mathrm{sd}=, 752)$. En menor medida, consideraron que mejoraron otras capacidades como el uso de la lengua extranjera ( $M=2,59$; sd=1,004), hablar en público $(M=3,04$; sd=,886) o su capacidad crítica y autocrítica ( $M=3,04 ;$ sd=,791).

Por otra parte, la formación académica se presentó como un factor determinante en los procesos de construcción e identificación vocacional de los deportistas de alto nivel ( $M=3,70$; sd=,513). Para los participantes en el estudio, la formación era un requisito indispensable para poder acceder al mercado de trabajo ( $\mathrm{M}=3,71$; $\mathrm{sd}=, 500)$, y por ello valoraron la importancia que tenía formarse a lo largo de la vida ( $M=3,75$; $\mathrm{sd}=, 436$ ) con la finalidad de adquirir conocimientos, competencias y habilidades para su desarrollo profesional ( $M=3,65$; sd=,550). De manera más precisa, en los procesos formativos, los deportistas encontraron un espacio donde aprender a tomar decisiones de manera eficaz ( $\mathrm{M}=2,43 ; \mathrm{sd}=, 800)$ y donde les informaron sobre cómo gestionar su trayectoria académica, vocacional y profesional ( $\mathrm{M}=2,53$; $\mathrm{sd}=, 809)$.

Si bien los datos obtenidos en el estudio apuntaron a la importancia que tenía la práctica deportiva y la formación académica como escenarios que permitieron a los deportistas de alto nivel definir su identidad vocacional, precisamente uno de los procesos más complejos al que se enfrentaron tuvo que ver con la compatibilización entre estas dos actividades. Una amplia mayoría de los participantes indicó que había simultaneado en algún momento de sus vidas los estudios y la práctica deportiva de alto nivel ( $M=3,28$; sd=,984). En este sentido, para el $72,0 \%$ de los deportistas, participar activamente y simultáneamente en estas dos importantes actividades para su desarrollo vocacional y profesional podía poner en riesgo, por sus elevadas exigencias, la continuidad y permanencia en alguna de ellas. Por ello, los deportistas buscaron estrategias para mantener una relación efectiva entre deporte y estudios, ya que, desde su perspectiva, la formación y la actividad deportiva constituían importantes factores para su trayectoria vital.

Para el 80,1\% de la muestra participante en este estudio, esta situación se debía principalmente a las altas demandas de estas actividades y a la falta de tiempo. Para superar estas dificultades, Los deportistas intentaban conciliar de manera equilibrada la dedicación a las distintas actividades (figura III). 


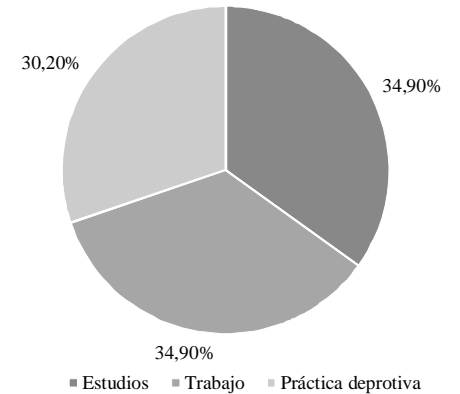

Figura III 3. Conciliación entre las diferentes actividades del deportista

Otro de los elementos que contribuyó a que los deportistas pudieran alternar las actividades académicas y deportivas, fue el apoyo que recibieron de personas allegadas, como eran familiares $(M=3,48$; $\mathrm{sd}=, 755)$, amigos $(\mathrm{M}=3,35$; $\mathrm{sd}=, 673)$, otros deportistas $(\mathrm{M}=3,30$; $\mathrm{sd}=, 777)$ y entrenadores $(\mathrm{M}=2,99 ; \mathrm{sd}=1,013)$. No obstante, resultó significativo que los orientadores académicos ( $\mathrm{M}=1,40$; sd=,704), los representantes deportivos ( $\mathrm{M}=1,34$; sd=,738) o los psicólogos deportivos ( $\mathrm{M}=1,37 ; \mathrm{sd}=, 722)$ no fueran apoyos significativos para los procesos de simultaneidad en los que participaron los deportistas de alto nivel. Además de estas ayudas, el 59,5\% de los encuestado indicó tener algún tipo de ayuda proveniente de distintas instituciones deportivas (Consejo Superior de Deportes, Consejería de Deportes, Clubes deportivos, marcas y representaciones deportivas, etc.) para facilitar los procesos de armonización.

Otro de los aspectos fundamentales que se incluyó en el modelo estaba relacionado con la información y conocimiento que tenían los deportistas de alto nivel sobre el mundo profesional. En este sentido, destacó que un reducido número de deportistas de alto nivel tuviera un conocimiento preciso sobre las características básicas del mercado labo$\operatorname{ral}(\mathrm{M}=2,40 ; \mathrm{sd}=, 577)$. De manera más específica, conocían las salidas profesionales que estaban vinculadas a las competencias y conocimientos que tenían adquiridos ( $\mathrm{M=2,38;} \mathrm{sd=,900),} \mathrm{los} \mathrm{requisitos} \mathrm{específicos}$ para acceder al trabajo que deseaban ( $\mathrm{M}=2,83$; $\mathrm{sd}=, 877)$ o las condiciones del puesto laboral al que le gustaría acceder $(\mathrm{M}=2,84 ; \mathrm{sd}=, 926)$. Por el contrario, tenían menos información sobre los centros de orientación profesional existentes ( $\mathrm{M}=2,04$; $\mathrm{sd}=, 784)$, las bolsas de empleo ( $=2,31$; $\mathrm{sd}=, 851)$, los tipos de contratos existentes ( $\mathrm{M}=2,37$; $\mathrm{sd}=, 917)$ o los índices de paro de las salidas profesionales preferentes $(\mathrm{M}=2,34$; sd=,941). A pesar de este escaso conocimiento, los deportistas de alto nivel consideraron que tenían las competencias y habilidades necesarias para desempeñar un trabajo cualificado (95,8\%), lo que se relaciona directamente con una elevada autoeficacia.

Uno de cada cuatro deportistas se encontraba trabajando en un puesto laboral relacionado con el deporte $(28,6 \%)$. Los relatos ofrecidos por los deportistas en las preguntas abiertas del cuestionario así lo reflejaron, cuando indicaron que se dedicaban actividades como: «entrenadora de gimnasia» (p167), «gerente de instalación deportiva» (p123), «médico del deporte» (p20), «periodista deportivo» (p66) o «técnico deportivo» (p182). Por su parte, el 71,4\% de personas que no dedicaron su actividad profesional al ámbito deportivo, desempeñaron sus funciones laborales en un amplio abanico de salidas como: «abogado» (p16), «comercial» (p143), «director financiero» (p84), «empresario» (p35), «funcionario de carrera» (p34), «informático» (p32), «maestro» (p28), «orientadora sociolaboral» (p75) o «profesor» (p102).

Los intereses profesionales de los deportistas de alto nivel se caracterizaron por ser trabajos que ofrecían un lugar y un ambiente de trabajo agradable $(M=3,56$; $s d=, 548)$, que fuera seguro y estable $(M=3,42$; $s d=, 669)$ y que les permitiera promocionar en la propia empresa ( $M=3,30 ;$ sd=,648). Por el contrario, aspectos como la posibilidad de movilidad geográfica ( $M=2,50 ;$ sd=,879) o el prestigio de la empresa no fueron muy valorados $(\mathrm{M}=2,90$; $\mathrm{sd}=, 726)$.

En cuanto a los procesos de toma de decisiones, los resultados obtenidos permitieron comprobar que los deportistas de alto nivel hacían uso de un estilo racional, mediante el cual valoraron las ventajas y desventajas de cada alternativa, recabaron información sobre cada una de las opciones, analizaron la adecuación de sus competencias, habilidades y conocimientos a sus deseos personales, etc. De manera más precisa, valoraron las consecuencias positivas y negativas $(\mathrm{M}=3,50$; $\mathrm{sd}=, 552$ ), estudiaron con detenimiento las distintas alternativas a las

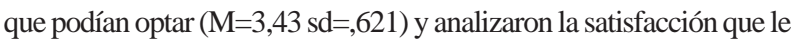
podía ofrecer ( $\mathrm{M}=3,38 ; \mathrm{sd}=, 654)$. También destacaron la importancia de otros aspectos en este proceso de elección, como fueron la valoración de los riesgos ( $M=3,29$; sd=,648) y el análisis del ajuste entre las competencias, habilidades y conocimientos y las expectativas de resultado esperadas y las preferencias personales ( $\mathrm{M}=3,26$; $\mathrm{sd}=, 655)$.

Finalmente, destacar que los deportistas de alto nivel tuvieron un alto nivel de dominio de las distintas competencias de adaptabilidad $(\mathrm{M}=3,28 ; \mathrm{sd}=0,38)$. La información obtenida reveló que los deportistas de alto nivel se caracterizaban por asumir las responsabilidades de sus propias acciones ( $\mathrm{M}=3,59$; $\mathrm{sd}=, 514)$, aprendían nuevas habilidades y capacidades para afrontar las tareas que se le propusieran $(M=3,57$; $\mathrm{sd}=, 518)$, aportaban soluciones creativas e innovadoras a distintos retos $(\mathrm{M}=3,45 ; \mathrm{sd}=, 530)$, planificaban y construían un plan de vida para lograr sus metas personales $(\mathrm{M}=3,04$; $\mathrm{sd}=, 717)$ y se preparaban a lo largo de la vida para su desarrollo profesional ( $\mathrm{M}=3,35$; $\mathrm{sd}=, 598)$.

\section{Discusión}

Tras el análisis teórico y empírico realizado, la conclusión general a la que hemos llegado es que, la definición vocacional y la construcción del proyecto profesional y vital de los deportistas de alto nivel es un complejo proceso multifactorial. En concreto, y rescatando la propuesta de Lent, Brown y Hackett (1994) y Lent, Brown y Hackett (2000), las variables de tipo contextual y cognitivo determinan la conducta vocacional del deportista de alto nivel. Efectivamente, y coincidiendo con el marco conceptual life-designing propuesto por Savickas, Nota, Rossier, Dauwalder et al. (2009) y el modelo del desarrollo de Wylleman y Lavalle (2004), se trata de un proceso holístico de identificación personal y vocacional que surge de la participación del deportista en diferentes contextos (deportivos, sociales, académicos, vocacionales, etc.).

Los resultados obtenidos han revelado que existen dos dimensiones básicas que permiten al deportista de alto nivel adquirir conocimientos, competencias y habilidades que resultan de interés para su desarrollo vocacional: deporte y formación. En consonancia con estos hallazgos, los trabajos de Garcés y Carlin (2011), Álvarez y López (2012) y Álvarez et al. (2014) destacan la importancia de la participación en actividades deportivas y académicas para los procesos de construcción de la identidad vocacional.

Sin embargo, de los resultados obtenidos se desprende que, los deportistas de alto nivel, tienen dificultades para mantener una relación efectiva entre estas dos actividades (Guilleetal., 2002; Marsh \& Kleiman, 2003; Urbina \& Miranda, 2004; Grissom, 2005; García et al, 2008; Ruiz, Salinero \& Sánchez, 2008). Al igual que demostraron Torregrosa \& Mimbrero (1998), Nuviala \& Nuviala(2005), Puig \& Vilanova (2006), Macarro et al, (2010), López et al. (2015), esta situación se debe fundamentalmente a la falta de tiempo y a las elevadas exigencias que demandan la actividad deportiva y la formación académica. En este sentido, estamos de acuerdo con Márquez et al. (2003), García et al. (2008) y Álvarez y López (2012) cuando sugieren la necesidad de diseñar programas de tutorización personalizada dirigida a los deportistas de alto nivel, cuya finalidad prioritaria es la de facilitar los procesos de compatibilización entre estudios y deporte.

Por otra parte, los hallazgos del estudio revelaron que los deportistas de alto nivel, a lo largo de su trayectoria vital, no se preocuparon por tener un conocimiento preciso de las características del mercado laboral. Sin embargo, y en relación al concepto de autoeficacia definido por Lent et al. (1994), los deportistas percibían que tenían un conocimiento sobre las competencias y habilidades que tenían adquiridas y que podrían ser de utilidad para su futuro desarrollo profesional. De la misma manera, el 
procedimiento de toma de decisiones empleado por parte de los deportistas de alto nivel se basaba en un estilo racional, enfrentando lo que eran capaz de hacer (autoeficacia) con lo que esperaban conseguir (expectativas de resultado). De esta manera, la definición de los intereses y motivaciones vocacionales se asentaban sobre presupuestos de valoración y madurez.

Para finalizar, los datos encontrados en la investigación indican que los deportistas de alto nivel tienen un elevado dominio de las competencias de adaptabilidad propuestas por Savickas (2005) y Hartung et al. (2008). Es decir, tienen un adecuado proceso de planificación, toma de decisiones, exploración y resolución de problemas. Esto permite al deportista de alto nivel enfrentarse, de una manera acertada, a las distintas circunstancias que tendrán lugar a lo largo de su desarrollo vital y que estarán en estrecha consonancia con su desarrollo vocacional.

\section{Referencias}

Álvarez, A. (2011). Inserción laboral de los futbolistas de élite tras su retirada. International Journal of Sport Sciences, 1 (2), 115-128.

Álvarez, P., Pérez-Jorge, D, López, D., y González, A.I. (2014). Transición y adaptación a los estudios universitarios de los deportistas de alto nivel: la compleja relación entre aprendizaje y práctica deportiva. Revista Española de Orientación y Psicopedagogía, 25(2), 74-89.

Álvarez, P., y López, D.(2012). Armonización entre proceso de aprendizaje y práctica deportiva en universitarios deportistas de alto nivel. Cultura, Ciencia y Deporte, 7 (21), $201-212$.

Bandura, A. (1997). Self-efficacy: the exercise of control. New York: Freeman.

Bermejo, B. (2006). La formación a lo largo de la vida: exigencias sociolaboralesdesarrollo personal. Educar, 38, 15-32.

Blanco,A. (2009). El modelo cognitivo social del desarrollo de la carrera: revisión de más de una década de investigación empírica. Revista de Educación, 350, 423-445.

Caput- Jogunica, R., Curkovic, S., \& Bjelic, G. (2012). Comparative analysis: support for student - athletes and the guidelines for the universities in the Southeast Europe. Sport Science, 5 (1), 21- 26.

Danish, S.J., Petitpas, A.J., \& Hale, B.D. (1993). Life development intervention for athletes: life skills through sports. The counseling psychologist, July, 352-385.

Díaz, P., Buceta, J.M., \& Bueno, A.M. (2004). Situaciones estresantes y vulnerables a las sesiones deportivas: un estudio con deportistas de equipo. Revista de Psicología del Deporte, 13(1), 7- 25.

Fernández, A., Stephan, Y., \& Fouquereau, E. (2006). Assessing reasons for sport career termination: development of theAthletes' Retirement Decision Inventory (ARDI). Psychology of Sport and Excercise, 7, 407-421.

Garcés, E.J., \& Carlin, M.(2011). El proceso de retirada y ajuste vital en deportista de alto rendimiento. Sevilla:Wanceulen Editorial Deportiva.

García, M. (1990). Aspectos sociales del deporte. Una reflexión sociológica. Madrid:Alianza.

García, T., Leo, F.M., Martín, E., \& Sánchez, P.A. (2008). El compromiso deportivo y su relación con factores disposicionales y situacionales contextuales de la motivación. Revista Internacional de Ciencias del Deporte, 12 (IV), 45-58.

Ginzberg, E., Ginsburg, S.W., Axelrad, S., \& Herma, J.L. (1951). Occupational choice: an approach to a general theory. New York: Columbia University Press.

González, M., Álvarez, P., Cabrera, L. y Bethencourt, J.T. (2007). El abandono de los estudios universitarios: factores determinantes y medidas preventivas. Revista Española de Pedagogía, LXV (236), 71-85.

González, M.D., \& Bedoya, J. (2008). Después del deporte, ¿qué? Análisis psicológico de la retirada deportiva. Revista de Psicología del Deporte, 17(1), 61-69.

González, M.D., \& Torregrosa, M. (2009).Análisis de la retirada de la competición de élite: antecedentes, transición y consecuencias. Revista Iberoamericana de Psicología del Ejercicio y del Deporte, 4(1), 93-104.

Grissom, J.B.(2005). Aptitud física y rendimiento académico. California: PubliCE Premium.

Guille, E., Sarrazin, P., Carperten, P.J., Trouilloud, D., \& Cury, F.(2002). Predicting persistence or withdrawal in female handballers with social exchange theory. International Journal of Psychology, 37(2), 93-104.

Hartung, P.J., Porfeli, E.J., \& Vondracek, F.W. (2008). Career adaptability in childhood. The Career Development Quarterly, 57, 63-74.

Holland, J.L. (1973). Making vocational choices: a theory of vocational personalities and work environments. Englewood Cliffs: Prentice-Hall.

Krumboltz, J.D. (1979). Asocial learning theory of career decision making. EnA.M. Mitchell, G.B. Jones, \& J.D. Krumboltz (Eds.), Social learning and career decision making (19-49). Cranston: Carroll Press.

Lent, R.W., \& Hackett, G (1987). Career self-efficacy: empirical status and future directions. Journal of Vocational Behavior, 30, 347-382.

Lent, R.W., Brown, S., \& Hackett, G. (1994). Toward a unifying social cognitive theory of career and academic interest, choice, and performance. Journal of Vocational Behavior, 45, 79-122.

Lent, R.W., Brown, S., \& Hackett, G (2000). Contextual supports and barriers to career choice: a social cognitive analysis. Journal of Counseling Psychology, 47(1), 36-49.

Lent, R.W., Hill, C.E., \& Hoffman, M.A. (2003). Development and validation of the counselor activity self-efficacy scales. Journal of Counseling Psychology, 50 (1), 97-108.

López, C., Barriopedro, M., Conde, E., Sánchez, J., Ubago, E., \& Gallardo, L. (2015).Análisis de las barreras percibidas por los deportistas de élite españoles para acceder a los estudios. Cuadernos de Psicología del Deporte, 15(1), 265274.

Macarro, J., Romero, C., \& Torres, J. (2010). Motivos de abandono de la práctica de actividad físico - deportiva en los estudiantes de bachillerato de la provincia de Granada. Revista de Educación, 353, 495-519.

Márquez, S., Salguero,A., \& Tuero, C. (2003). Adaptación española del cuestionario de causas de abandono en la práctica deportiva: validación y diferencias de género en jóvenes nadadores. Revista Digital, 8(56).

Marsh, H.W., \& Kleiman, S. (2003). School athletic participation: mostly again with little pain. Jorunal of Sport and Exercise Psychology, 25, 205-228.

McMillan, E., \& Schumacher, S. (2005). Investigación educativa (5a Edición). Madrid: Pearson.

Nuviala,A., \& Nuviala, R. (2005). Abandono y continuidad de la práctica deportiva escolar organizada desde la perspectiva de los técnicos de una comarca aragonesa. Revista Internacional de Medicina y Ciencias de la Actividad física y el Deporte, 5 (20), 295-307.

Puig, N., \& Vilanova, A. (2006). Deportistas olímpicos y estrategias de inserción laboral: propuesta teórica, método y avance de resultados. Revista Internacional de Sociología, LXIV(44), 63-83.

Roe,A.(1954).Anew classification of occupations. Journal of Counselling Psychology, $1,215-220$.

Ruiz, G, Salinero, J.J., \& Sánchez, F. (2008). Valoración del perfil sociodemográfico del triatleta: el ejemplo de Castilla - La Mancha: nivel de implicación y entorno. Apunts de Educación Física y Deportes, 2, 5-14.

Savickas, M.L. (2005). The theory and practice of career construction. En S.D. Brown y R.W. Lent (Eds.), Career Development and Counseling: Putting theory and research to work (42-70). New Jersey: John Wiley \& Sons, Inc.

Savickas, M.L., Nota, L., Rossier, J., Dauwalder, J., Duarte, M.E., Guichard, J., Soresi, S., Esbroeck, R.V., \& Vianen, A.E.M. (2009). Life designing: a paradigm for career construction in the $21^{\text {st }}$ century. Journal of Vocational Behavior, 75, 239-250.

Savickas, M.L., \& Porfeli, E.J.(2012). CareerAdapt-Abilities Scale: construction, reliability, and measurement equivalence across 13 countries. Journal of Vocational Behavior, 80, 661-673.

Solà, J.(2015). Educación secundaria y deporte de alta dedicación: estudio cualitativo de modelos de compaginación. Retos, Nuevas tendencias en Educación Física, Deportes y Recreación, 28, 54-60.

Super, D.E., Crites, J.O., Hummel, R.C., Moser, H.P., Overstreet, P.L., \& Warnath, C.F.(1957). Vocational development: a framework for research. Nueva York: Columbia University.

Tiedeman, D.V., \& O’Hara, R.P.(1963). Career development: choice and adjustment. New York: College Entrance Examination Board.

Torregrosa, M., \& Mimbrero, J. (1998). Perfiles profesionales de deportistas olímpicos. Barcelona: Fundación Barcelona Olímpica.

Torregrosa, M., Sánchez, X., \& Cruz, J. (2004). El papel del psicólogo del deporte en el asesoramiento académico-vocacional del deportista de élite. Revista de Psicología del Deporte, 13(2), 215-228.

Urbina, S., \& Miranda, N. (2004). Niños deportistas tiene mejor rendimiento escolar. Extraído el 24 de mayo de 2017 de http://www.reduc.cl/raes.nsf/ PgRecortesPorAutor?OpenPage\&Start=21.7\&Count=30\&Expand $=21.7$

Valiente, G. (2000). Writing self-efficacy and gender orientation. Adevelopmental perspective. Atlanta: Emory University.

Wylleman, P., Alfermann, D., \& Lavallee, D. (2004). Career transitions in sport: European perspectives. Psychology of Sport and Exercise, 5, 7-20.

Wylleman, P., De Knop, P., Ewing, M., \& Cumming, S. (2000). Transitions in youth sport: a developmental perspective on parental involvement. En D. Lavallee y P. Wylleman (Ed.) Career transitions in sport: international perspectives (143-160). Morgantown: Fitness Information Technology.

Wylleman, P., Vanden, Y., De Knop, P., Sloore, H., \& De Martelaer, K. (1995). Elite young athletes, parents, and coaches: relationships in competitive sports. The $1^{\text {st }}$ Bath Sports Medicine Conference. Bath: United Kingdom.

Wylleman, P., \& De Knop, P. (1997). The role and influence of the psycho-social environment on the career transitions of student- athletes. $2^{\text {nd }}$ Annual Congress of the European College of Sports Science. Copenhagen, Denmark.

Wylleman, P., \& Lavallee, D. (2004). Adevelopmental perspective on transitions faced by athletes. En M. Weiss (Ed.), Developmental sport and exercise psychology: a lifespan perspective (507-527). Morgantown: Fitness Information Technology. 